\title{
Does Ultrasounding Children Referred for Appendicitis Aid the Choice to Operate?
}

\author{
Matthew LM Jones ${ }^{1,2,3 *}$, Amy L Sweeny ${ }^{4}$ and Richard J Thompson ${ }^{1}$ \\ ${ }^{1}$ Department of Paediatric Surgery, Gold Coast University Hospital, Australia \\ ${ }^{2}$ Department of Paediatric Surgery, The Royal Children's Hospital, Australia \\ ${ }^{3}$ F Douglas Stevens' Research Laboratory, The Murdoch Children's Research Institute, Australia \\ ${ }^{4}$ Emergency Medicine Foundation; Gold Coast University Hospital, Australia
}

Submission: February 29, 2020 Published: April 06, 2020

*Corresponding author: Matthew Lachlan Markley Jones, Department of Paediatric Surgery, The Royal Children's Hospital, Melbourne, Australia

\begin{abstract}
Purpose: To determine whether the use of ultrasound (USS) assists in the diagnosis of appendicitis in paediatric patients.

Method: A retrospective study of patients <14 years, referred to paediatric surgery with possible appendicitis from Jul 2015 to Nov 2016. Groups were based on the decision to admit with or without USS and their Alvarado scores (AS). Diagnosis was confirmed by histopathology. Outcomes were missed appendicitis, negative appendicectomy rate (NAR), and specificity of diagnosis.

Results: 234 patients were included. 126 (54\%) underwent appendicectomy and 102 (44\%) had appendicitis. USS was performed in 163 patients (70\%) and was $83 \%$ sensitive and $87 \%$ specific. Clinical diagnosis had greater specificity with USS $88 \%$ (CI 95\%, 80-93) than without USS 59\% (39-78). There was no significant difference in the NAR with USS (18\%) and without (20\%). All 29 patients AS 9-10 had appendicitis of these $15(52 \%)$ had USS prior to theatre. None of the 30 patients AS 0-3 had appendicitis. In the group of 98 patients AS 6-8, those 59 who had an USS had a NAR of $2.86 \%$ and specificity of $96 \%$ (80-100), compared to NAR of $20 \%$ and specificity of $36 \%$ (11-69) in the 39 patients without.

Conclusion: USS aids diagnostic accuracy through increasing the specificity, it does not benefit in AS $\geq 9$ and $\leq 3$ where the diagnosis is clinically evident. Consideration should be made to reduce these USS to lessen referral delay, hospital expense and misleading clinical decisionmaking. USS appears most useful in patients AS 6-8, with improved outcomes identified.

Keywords: Ultrasound; Histopathology; Appendectomy; Alvarado; Diagnosis; Appendicitis; Children; Verbalise; Hopping; Investigations; Neutrophils; Anorexia; Abdominal; Surgery; Paediatric; Sonographer; Spreadsheets; Radiology; Registrars; Consultants

Abbreviations: NAR: Negative Appendicectomy Rate; NPV: Negative Predictive Value; PPV: Positive Predictive Value; LOS: Length of Stay; GCUH: Gold Coast University Hospital; RIF: Right Iliac Fossa
\end{abstract}

\section{Introduction}

Appendicitis remains the most common condition in children requiring emergency abdominal surgery [1]. The key to successful outcome in patients affected is early diagnosis with appendicectomy performed prior to gangrene or perforation developing. The ability to diagnoses this condition however becomes increasingly difficult in children, due to non-specific presentations, inability to verbalise symptoms, and the child's apprehension to examination and investigations [2]. Because of this investigation need to be targeted with justifiable outcomes. The clinical features of appendicitis vary with age, with the most frequent being tenderness of the right iliac fossa (RIF), difficulty walking, pain on hopping/cough, nausea, vomiting, anorexia and fevers. This condition is commonly associated with a raise in the number of white cells (in particular neutrophils) on a full blood count. Multiple scoring systems have been adapted to categorise these symptoms and findings into a 'likelihood of appendicitis', the most common being the Alvarado Score and the Paediatric Appendicitis Score [3] USS is an inexpensive and safe investigation as part of the work-up of appendicitis [4]. Its use in combination with Alvarado scores has been shown to increase the positive predictive value of diagnosis [5]. USS has the added advantage in the work-up of appendicitis of being capable of assessing ovarian pathology in females as part of the differential diagnosis. A large 
single institute study has recently been conducted identifying USS sensitivity for appendicitis as $97.1 \%$ and specificity of $94.8 \%$ [6]. USS however requires skill and experience of the sonographer, with a wide variance of accuracy seen between centres.

\section{Method}

\section{Design and participants}

This was a retrospective study performed at the Gold Coast University Hospital (GCUH), Queensland, Australia. Ethics approval was granted from the GCHHS HREC as a quality activity. Patients recruited were children aged under 14 years-old, who were referred to the Paediatric Surgery team at GCUH for 'query appendicitis' between the dates of 01 July 2015 - 30 Nov 2016 (17 months). Patient's details were collected from the monthly audit data spreadsheets. The patient's 'signs and symptoms' were recorded based on the paediatric or general surgical registrar's admission/review note, with investigations based on results on electronic laboratory results (AusLab) or medical imaging reports (impax) conducted at the time of review. USS was performed in the Emergency Department by trained sonographers and interpreted by radiology registrars and consultants (with reports signed-off by consultants).

\section{Outcomes}

Patients were able to be categorised based on their signs, symptoms and investigations within Alvarado scores. The Alvarado scores used included: unlikely appendicitis (AS 0-3); possible appendicitis (AS 4-5); likely appendicitis (AS 6-8); and clinical appendicitis (AS 9-10). All patients within the study had the presence or absence of the relevant criteria reported for the Alvarado Score to be assessed and all received blood tests while in ED for WCC. Patients were also grouped based on whether they received an USS prior to proceeding to theatre, or discharge home. The USS groupings included those suggestive of appendicitis (USS reported as 'positive'), those that were not suggestive of appendicitis (USS reported as 'negative', 'equivocal' or 'appendix not seen'), and those in who USS was not performed. USS were performed at either GCUH, Robina Hospital (the Gold Coast Health Service partner hospital), Tweed Heads Hospital, or private USS requested by the patients GP. USS were considered to be 'positive' where they states in the summary of the report "appendicitis", "early appendicitis", "findings in keeping with appendicitis", or "appendicitis with evidence of perforation". The diagnosis of appendicitis was based on histopathology in keeping with 'early appendicitis', 'acute appendicitis', 'acute suppurative appendicitis' or 'acute suppurative appendicitis with focal perforation'. Patients were deemed to not have appendicitis based on histopathology showing a 'normal appendix', or an appendix with 'No significant inflammatory infiltration'. Patients were also considered to have a normal appendix if alternative pathology was identified and a normal appendix seen and reported in the operative report, or who were discharged from hospital without theatre and did not return. No patients had primary antibiotic treatment for suspected appendicitis. The decision to proceed to theatre with a consent form stating "appendicitis", or "likely appendicitis" as the indication for surgery, was assessed as the surgeon's diagnosis of appendicitis.

\section{Statistical analysis}

Data was entered into an electronic spreadsheet (Microsoft Excel 2016, Microsoft Corporation, Seattle, WA, USA) where further statistical analysis was performed. Unpaired T-tests, and chi-squared were used to calculate statistical significant between demographics of groups. Primary statistical analysis included calculating the rate of Missed Appendicitis, the Negative Appendicectomy Rate (NAR), sensitivity, specificity, positive predictive value (PPV) and negative predictive value (NPV) of diagnosis for the whole cohort. The sensitivity and specificity of USS was also assessed. Confidence Intervals for sensitivity and specificity were calculated as exact Clopper-Pearson intervals. This analysis was then repeated on individual groups after separating the patients based on Alvarado Scores. These categories were agreed upon by authors and based upon scores abilities to rule in or out appendicitis $(0-3,9-10)$, and the balance of probability $(A S=5)$ [7]. The value of $p=0.05$ was used to assess for statistical significance.

\section{Results}

A total of 234 patients were assessed in this study. 126 (53.8\%) patients proceeded to theatre for presumed appendicitis, and 102 (43.6\%) had histopathology diagnostic of appendicitis. 2 patients had other pathology identified (1 Meckle's diverticulitis, 1 fimbrial cyst), and 22 patients had a normal appendix sent to pathology. The patient demography with comparison between patients who did or did not receive USS. Statistical significance is demonstrated between groups with a higher percentage of females within the USS group, and a higher AS for the 'No USS' group. There was no significant difference between age and length of stay (LOS). USS was performed in 163 (69.7\%) of participants with 62 patients undergoing USS supportive of appendicitis. 101 USS were not supportive of appendicitis, with the appendix not seen in 57 (35.0\% of USS performed), 22 USS reported as 'negative', and 22 USS reported as equivocal. Of the USS performed 18 were completed at private radiology practices prior to attending ED. In assessing the accuracy of clinical assessment with or without USS, there were no cases of 'missed appendicitis' in either group, subsequently sensitivity is calculated as $100 \%$ for all groups. The USS group had a significantly higher specificity of diagnosis $87.6 \%(79.8-93.2 ; 95 \% \mathrm{CI})$ compared to the non-USS groups $59.3 \%$ (38.8-77.6; 95\% CI). The NAR was found to be $20 \%$ and $18.3 \%$ respectively. Patients were then separated based on their Alvarado scores as shown in Figure 1. 29 patients had an AS 9-10. Of these, $100 \%$ proceeded to theatre with histopathology confirming appendicitis. 15 (52\%) had USS performed prior to proceeding to theatre, with 1 equivocal and 2 'not seen' results. 98 patients had AS 6-8, with 59 (60.2\%) of these patients receiving 
USS as part of their work-up. There was a significantly greater specificity of diagnosis in the USS group, 96\% (79.7-99.9; 95\% CI), than the non-USS group, $36.4 \%$ (10.9-69.2; 95\% CI). The NAR was $2.9 \%$ and $20 \%$ respectively. $63.3 \%$ of patients within this cohort had appendicitis. Of the 77 patients with an AS 4-5, 62 (80.5\%) received USS as part of their work-up. The NAR in this cohort is $50 \%$ with USS and $66.7 \%$ without USS. 24 (31.1\%) patients in this group were taken the theatre, with only $11(14.3 \%)$ having appendicitis. There was no significant differences in the clinical accuracy with or without USS in this cohort. 30 patients had AS $0-3$, of which none had appendicitis. 27 (90\%) underwent USS investigation, with 3 USS reports suggestive of appendicitis. 3 patients were taken to theatre ( 2 based on their USS report), with normal appendix on histopathology. The appendix was not seen on USS in $14(46.7 \%)$ of these patients. In the 22 patients with an USS scan reported as 'negative' for appendicitis, there were no cases of appendicitis. This counted for $13.5 \%$ of scans performed.

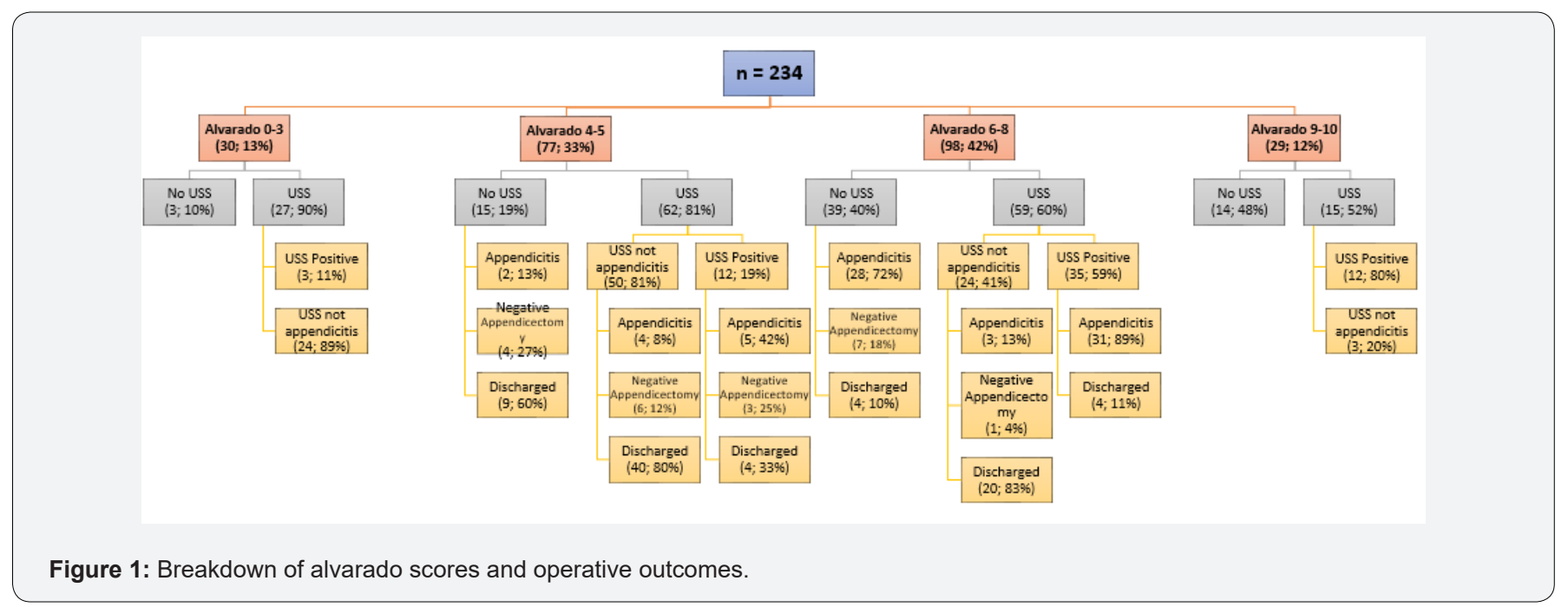

\section{Discussion}

Overall, it can be seen that the addition of USS as part of the clinical work-up of a patient with 'query appendicitis' does improve the clinical accuracy of diagnosis, as seen through greater specificity. This study suggests that USS alone would miss $17.2 \%$ of cases of appendicitis, and therefore results 'not suggestive of appendicitis' it remains important to keep in the context of the patient. This study suggests the accuracy of USS performed in a tertiary hospital, with joint adult and paediatric care, is not as sensitive or specific at diagnosing appendicitis as shown in larger single institute studies. The variability of USS accuracy is most likely attributable to the requirement of skilled ultra-sonographer to obtain adequate images, including subjective dynamic aspects of this study (ie. probe tenderness or non-compressibility). It I possible that centres which perform abdominal USS regularly on children would have greater technical capability to obtain these factors accurately. The break-down of patient cohorts within this study provides a basis for a more targeted use of USS, where its overall addition to clinical accuracy can be focussed. The cohort AS 6-8, seems to have the greatest addition to management through the use of USS. The reduction in NAR and significantly greater sensitivity of clinical diagnosis in the USS group, suggests the investigation is of most use in this cohort. Within the cohort of AS 9-10, the clinical picture seems to be convincing enough to justify surgical review and consideration for theatre, without the need for USS performed prior. USS in this cohort did not change the management for any patients, and serves as an unnecessary cost, delay and potential further discomfort and distress to the patient. The absence of any cases of confirmed appendicitis within the cohort of AS 0-3, suggests a clinical score this low alone is strongly suggestive against appendicitis. Subsequently USS would seem of little use, and clinical concern for a patient within this cohort could be arguably better managed through observation and interval clinical assessment rather than further investigation. Given the high proportion of appendix's not seen, equivocal or positive scan results, it does not seem to add much for the argument of obtaining an USS to reassure patient's or parents. Outside the scope of this study is the role of USS in diagnosing alternative abdominal pathology in children. Diagnosis such as ovarian torsion (and other ovarian pathology), intussusception and Meckle's diverticulitis remain important considerations in the work-up of paediatric patients with abdominal pain. It would be suggested that the use of USS for these differentials should be considered by the treating clinician. The limitations of this study include its retrospective nature, and recruitment bias secondary to this. This is demonstrated through the statistically significant demographics represented by the USS and non-USS group. The higher percentage of females receiving USS is likely secondary to the possibility of ovarian pathology during their diagnostic work-up. The trend towards an average lower AS in the USS group, likely corresponds to less clinical certainty in the diagnosis, and 
investigating for alternatives. The use of the Alvarado Score for the clinical assessment, as opposed to Paediatric Appendicitis Score, is a further limitation, with studies demonstrating greater accuracy with the PAS in paediatric population.

\section{References}

1. Glass CC, Rangel SJ (2016) Overview and diagnosis of acute appendicitis in children. Semin Pediatr Surg 25(4): 198-203.

2. Doria AS (2009) Optimizing the role of imaging in appendicitis. Pediatric Radiology 39(2): 144-148.

3. Bachur RG, Callahan MJ, Monuteaux MC, Rangel SJ (2015) Integration of ultrasound findings and a clinical score in the diagnostic evaluation of pediatric appendicitis. J Pediatr 166(5): 1134-1139.
4. Bachur RG, Hennelly K, Callahan MJ, Monuteaux MC (2012) Advanced Radiologic Imaging for Pediatric Appendicitis, 2005-2009: Trends and Outcomes. The Journal of Pediatrics 160(6): 1034-1038.

5. Zouari M, Jallouli M, Louati H, Kchaou R, Chtourou R, et al. (2016) Predictive value of C-reactive protein, ultrasound and Alvarado score in acute appendicitis: a prospective pediatric cohort. The American Journal of Emergency Medicine 34(2): 189-192.

6. Cundy TP, Gent R, Frauenfelder C, Lukic L, Linke RJ, et al. (2016) Benchmarking the value of ultrasound for acute appendicitis in children. J Pediatr Surg 51(12): 1939-1943.

7. Ebell MH, Shinholser J (2014) What are the most clinically useful cutoffs for the Alvarado and Pediatric Appendicitis Scores? A systematic review. Ann Emerg Med 64(4): 365-372. 